Research Article

\title{
Power Control for Passive QAM Multisensor Backscatter Communication Systems
}

\author{
Shengbo Hu, ${ }^{1,2}$ Jinrong Mo, ${ }^{1,2}$ Tingting Yan,,2 and Yanfeng Shi ${ }^{1,2}$ \\ ${ }^{1}$ Institute of Intelligent Information Processing, Guizhou Normal University, Guiyang 550001, China \\ ${ }^{2}$ Center for RFID and WSN Engineering, Department of Education, Guizhou Normal University, Guiyang 550001, China \\ Correspondence should be addressed to Jinrong Mo; 935472997@qq.com
}

Received 25 April 2017; Accepted 3 October 2017; Published 27 December 2017

Academic Editor: Nashwa El-Bendary

Copyright (C) 2017 Shengbo Hu et al. This is an open access article distributed under the Creative Commons Attribution License, which permits unrestricted use, distribution, and reproduction in any medium, provided the original work is properly cited.

\begin{abstract}
To achieve good quality of service level such as throughput, power control is of great importance to passive quadrature amplitude modulation (QAM) multisensor backscatter communication systems. First, we established the RF energy harvesting model and gave the energy condition. In order to minimize the interference of subcarriers and increase the spectral efficiency, then, the colocated passive QAM backscatter communication signal model is presented and the nonlinear optimization problems of power control are solved for passive QAM backscatter communication systems. Solutions include maximum and minimum access interval, the maximum and minimum duty cycle, and the minimal RF-harvested energy under the energy condition for node operating. Using the solutions above, the maximum throughput of passive QAM backscatter communication systems is analyzed and numerical calculation is made finally. Numerical calculation shows that the maximal throughput decreases with the consumed power and the number of sensors, and the maximum throughput is decreased quickly with the increase of the number of sensors. Especially, for a given consumed power of sensor, it can be seen that the throughput decreases with the duty cycle and the number of sensors has little effect on the throughput.
\end{abstract}

\section{Introduction}

Since the advent of backscatter communication, it has been widely used in the passive RFID (radio frequency identification) system due to its low cost and low power $[1,2]$. Similarly, using the principle of the backscatter communication, the passive sensor node transmitter can be simplified into a transistor connected to the antenna. This will greatly reduce the cost and power consumption of wireless communication systems. For example, scholars from the University of Washington [3] researched the WISP (wireless identification and sensing platform) wireless smart sensor systems which can harvest RF energy based on backscatter communication. Now, PBC (passive backscatter communication) has been applied in these fields of smart car, wireless temperature measurement, the biological signal acquisition [4-9], and so forth. Undoubtedly, the study and application of passive backscatter communication will attract more and more attention.

In passive backscatter communication systems, sensor nodes need to harvest RF energy from the transceivers or reader and use it to recharge their finite energy storage capacity as shown in Figure 1. However, the unpredictable environments such as the channel, the number of sensors, and challenges of the RF energy harvesting make the activities of the sensors difficult, including sensing, processing, and communicating by nodes with a given rate. So, an efficient energy management is necessary in order to guarantee sensors' activities and a QoS (quality of service) of backscatter communication [10-12].

In fact, the mode using RF energy harvesting as a supplement to the finite energy storage capacity is called energy neutral operation [13]. In this mode, for such RF energy harvesting nodes with ideal finite energy storage capacity, the condition for energy neutral operation should be satisfied for all nonnegative values of $\tau$ :

$$
\int_{0}^{\tau} P_{\mathrm{c}}(t) d t \leq \int_{0}^{\tau} P_{\mathrm{RF}}(t) d t+B_{0}
$$

where $P_{\mathrm{RF}}(t)$ is the harvested RF power at time $t, P_{\mathrm{c}}(t)$ is the consumed power by nodes at that time, $B_{0}$ is the initial 


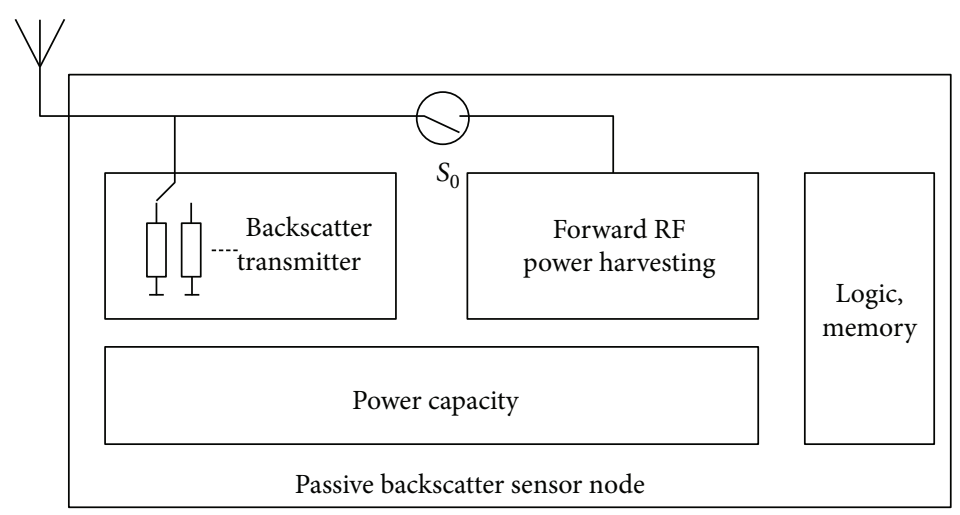

Figure 1: Passive backscatter sensor node.

energy stored in the ideal capacity, and $\tau$ is the runtime. So, the goal of power control for passive backscatter communication is to maximize QoS level under condition of energy neutral operation.

For implementing multisensor backscatter communication systems, subcarrier modulation can be used for each sensor [14]. This leads to interference among sensors. In order to minimize such interference and increase spectral efficiency, modulations including QAM are used to improve data throughput [15-17]. It shows that it is feasible to use load-dependent scattering for yielding a simple QAM backscatter [15, 17]. In this paper, we focus on colocated multisensor QAM backscatter communication, showing that power control is important for the backscatter communication systems to achieve good QoS level via analysis and simulation. We introduce a related work in Section 2 and describe a system model in Section 3. In Section 4, we present the performance of the backscatter communication systems. And we analyze the impact of power control on the throughput of systems in Section 5. Finally, we provide some concluding remarks in Section 6.

\section{Related Work}

Several papers have mainly proposed power control on nodes using energy harvesting, because power control of energy harvesting determines the level of connectivity of backscatter communication as well as the achievable QoS of the systems. An RF-powered transmitter that supports $915 \mathrm{MHz}$ downlink and $2.45 \mathrm{GHz}$ uplink bands is designed in [18]. In [19], the paper computes the minimal number of sinks required to keep the network connected and to satisfy the required constraints.

There exist attractive applications of the RF-powered devices such as wireless body networks. Benefiting from RF energy harvesting, some low-power devices can achieve real-time work-on-demand power from RF sources, which further enables a battery-free circuit with reduced size [20]. The body devices that implement high efficiency can be found in [21, 22]. For the multisensor system, we can refer [23]. The authors address the problem of developing energy-efficient transmission strategies for body sensor networks with energy harvesting [24]. And the authors evaluate the impact of transmit power control on the usefulness of a multisink WSN-Heap using energy harvesting, deployed in uniform string topology for railway track monitoring [25].

\section{System Models}

3.1. RF Energy-Harvesting Model. The RF energy harvester is composed of an antenna, impedance matching circuit, rectifier, and voltage multiplier as shown in Figure 2. The efficiency $\eta$ of the RF energy harvester is defined as follows:

$$
\eta=\eta_{1} \eta_{2} \eta_{3}=\frac{P_{\mathrm{eh}}}{P_{\mathrm{in}}}
$$

where $\eta_{1}, \eta_{2}$, and $\eta_{3}$ are the efficiencies of the receiving antenna, impedance transformation network, and the rectifier, respectively, $P_{\text {eh }}$ is the output DC power and the meaning of eh is energy harvesting, and $P_{\text {in }}$ is the input RF power.

In Figure 2, $Y$-stage voltage multiplier circuits are used to promote the output voltage. So, the upper limit of the steadystate output voltage $V_{\mathrm{dd}}$ is increased by a factor $Y$ and can be written as [26]

$$
V_{\text {dd }}=2 Y\left(V_{\text {peak }}-V_{\text {on }}\right) \text {, }
$$

where $V_{\text {peak }}$ is the peak voltage of the signal at the input of the view of the $Y$-stage voltage multiplier circuits and $V_{\text {on }}$ is the turn-on voltage of the diode.

Now, let us consider the minimum voltage for nodes to operate. Assuming, the harvesting power from the transceivers or reader is $P_{\mathrm{eh}}(t)$ and the consumed power by nodes at that time is $P_{c}(t)$. When the power made available by the transceivers or reader is insufficient for continuous operation, that is, $P_{\mathrm{eh}}(t)<P_{\mathrm{c}}(t)$, a capacity must be used to store RF energy until enough RF energy exists to complete an access. This affects the backscatter communication QoS level. Under these circumstances, the capacitor must be charged unless the stored energy $E_{\text {stored }}$ is greater than or equal to the access energy $E_{\text {access }}$, where $E_{\text {access }}$ is the energy required for each access.

The stored energy $E_{\text {stored }}$ can be written as

$$
E_{\text {stored }}=\frac{1}{2} C\left(V_{\mathrm{dd}}^{2}-V_{\text {node }}^{2}\right) \text {, }
$$




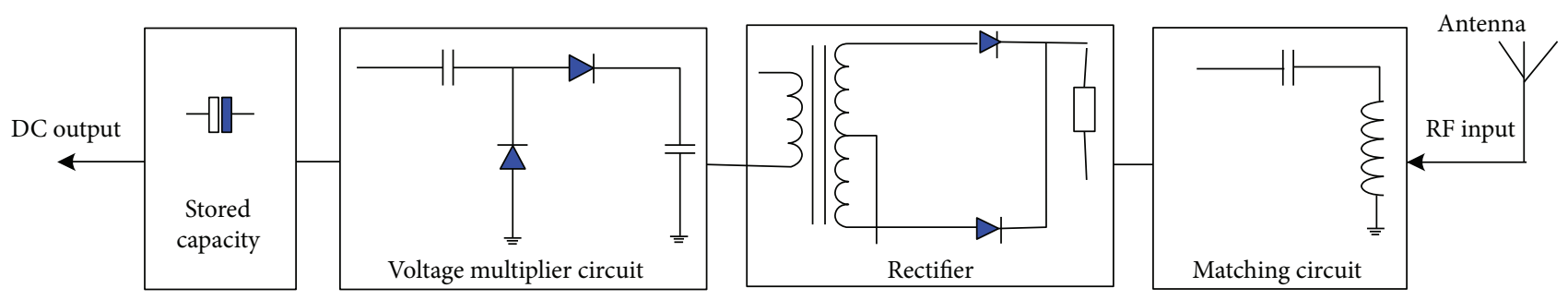

(a)

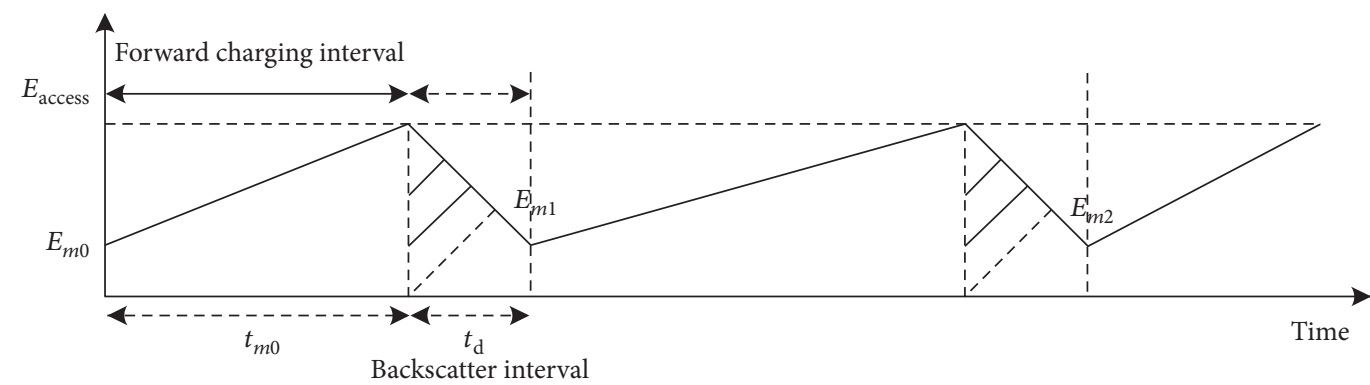

(b)

FiguRE 2: RF energy-harvesting model.

where $C$ is the value of energy harvesting circuit output capacity and $V_{\text {node }}$ is the voltage level at which the node operates. And the access energy $E_{\text {access }}$ can be written as

$$
E_{\text {access }}=\int_{T_{0}}^{T_{0}+T_{\text {access }}} P_{c} d t=V_{\text {node }} \int_{T_{0}}^{T_{0}+T_{\text {access }}} I_{\text {access }} d t,
$$

where $\left[T_{0}, T_{0}+T_{\text {access }}\right]$ is the access interval and $I_{\text {access }}$ is the time-varying current draw of the node during each access.

Hence, the minimum voltage for nodes to operate is solved as

$$
V_{\mathrm{dd}}^{\min }=\sqrt{\left(\frac{2 E_{\text {access }}}{C}\right)+V_{\text {node }}^{2}} .
$$

Once $V_{\mathrm{dd}}<V_{\mathrm{dd}}^{\min }$, the node can not operate.

3.2. Energy Condition for Node Operating. Backscatter communication links include forward link and backscatter link. While accessing, the node harvests RF energy from the transceivers or reader firstly by forward link. Once $V_{\text {dd }} \geq V_{\mathrm{dd}}^{\min }$, that is, $E_{\text {stored }} \geq E_{\text {access }}$, the node draws power to carry out its operation and sends the sensor data to the transceivers or reader by backscatter link. These can be defined as an energy model as given in Figure 2. Each access interval comprises a charging phase and backscatter communication phase. In Figure 2, while accessing at time $T_{0}$, the capacitor $C$ charges unless the stored energy is $E_{\text {stored }} \geq E_{\text {access }}$ at time $T_{0}+T_{c}$. Then, the node sends data by backscatter link. Once $E_{\text {stored }}<E_{\text {access }}$ at time $T_{0}+T_{\mathrm{c}}+T_{d}$, the node stops operating and waits next access. Let $E_{\text {eh }}=$ $B_{0}+\eta \int_{T_{0}}^{T_{0}+T_{\mathrm{c}}}\left[P_{\mathrm{eh}}(t)-P_{\mathrm{c}}(t)\right]^{+} d t$, the condition for energy neutral operation should be satisfied in the access interval $\left[T_{0}, T_{0}+T_{\text {access }}\right]$ :

$$
E_{\mathrm{eh}}-\int_{T_{0}+T_{\mathrm{c}}}^{T_{0}+T_{\mathrm{c}}+T_{d}}\left[P_{\mathrm{c}}(t)-P_{\mathrm{eh}}(t)\right]^{+} d t \geq 0
$$

where $\eta \in[0,1]$ is the charging rate, $B_{0}$ is the initial energy stored in the capacity, and the rectifier function $[x]^{+}$is defined as follows:

$$
[x]^{+}= \begin{cases}x, & x \geq 0 \\ 0, & x<0 .\end{cases}
$$

Meanwhile, the limitation of capacity size requires the constraint to be satisfied as follows:

$$
E_{\mathrm{eh}}-\int_{T_{0}+T_{\mathrm{c}}}^{T_{0}+T_{\mathrm{c}}+T_{d}}\left[P_{\mathrm{c}}(t)-P_{\mathrm{eh}}(t)\right]^{+} d t \leq B,
$$

where $B$ is the maximum energy that can be stored in the capacitor.

Hence, the energy conditions for the node operating include (7), (9), and (10). And (10) is given as follows:

$$
B_{0}+\eta \int_{T_{0}}^{T_{0}+T_{\mathrm{c}}}\left[P_{\text {eh }}(t)-P_{\mathrm{c}}(t)\right]^{+} d t \geq E_{\text {task }}
$$

where $E_{\text {task }}$ is the minimum energy required for the node to operate.

For simplicity, assuming the consumed power by the node with forward link is $P_{\mathrm{fc}}$, the consumed power by the sensor with backscatter link is $P_{\mathrm{bc}}\left(P_{\mathrm{fc}}<P_{\mathrm{bc}}\right)$ and $P$ is 


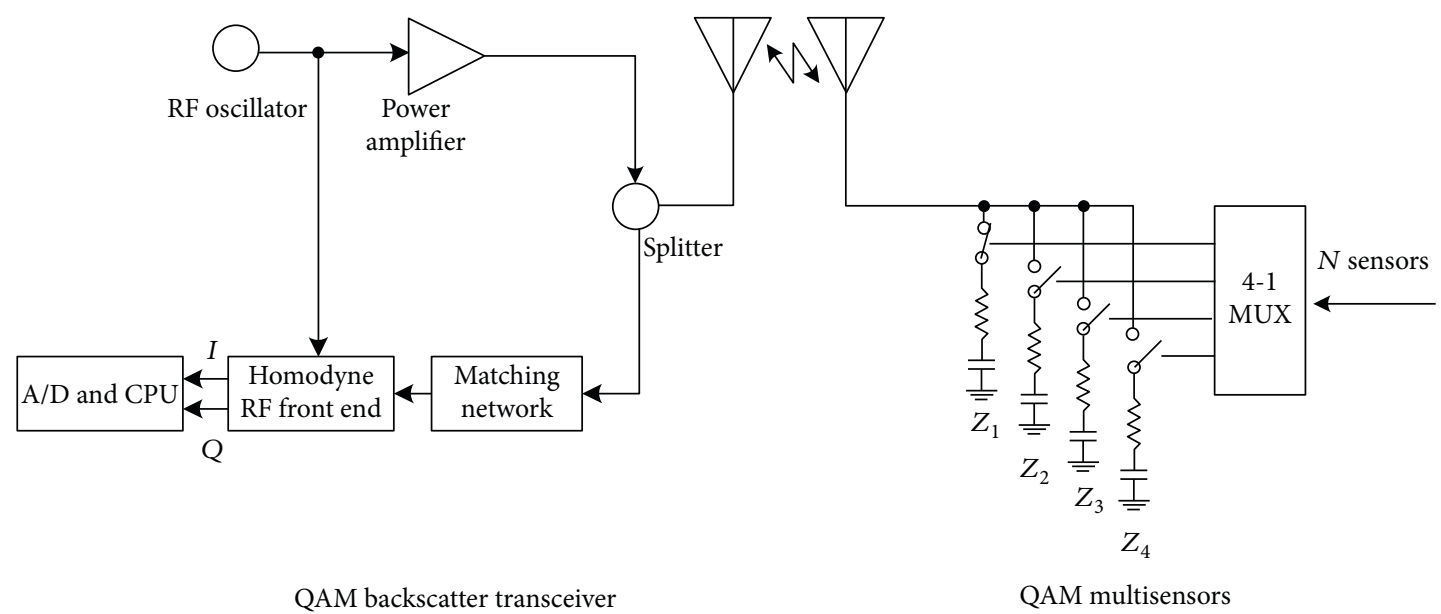

Figure 3: Passive QAM backscatter multisensor communication systems.

the harvested power, then the energy condition for node operating is given as follows:

$$
\begin{aligned}
B_{0}+\eta T_{\mathrm{c}}\left(P-P_{\mathrm{fc}}\right)-T_{d} P_{\mathrm{bc}} & \geq 0, \\
B_{0}+\eta T_{\mathrm{c}}\left(P-P_{\mathrm{fc}}\right)-T_{d} P_{\mathrm{bc}} & \leq B, \\
B_{0}+\eta T_{\mathrm{c}}\left(P-P_{\mathrm{fc}}\right) & \geq E_{\text {access }} .
\end{aligned}
$$

\section{Performance Analysis}

4.1. Colocated QAM Multisensor Backscatter Signal. Typically, backscatter communication systems use singlefrequency continuous wave transmission waveforms for accessing. The resulting complex signal from the node received at the transceivers or reader is given as

$$
r(t)=\sqrt{2 E \alpha} m(t) \exp \left[j\left(\omega_{c} t+\varphi\right)\right]+n(t),
$$

where $E, \omega_{c}$, and $\varphi$ are the peak power, the angular frequency, and the phase of the carrier signal, respectively, $n(t)$ is AWGN (additive white Gaussian noise), and $m(t)$ is the message signal to be transmitted.

To improve spectral efficiency, the use of QAM opens up many new avenues for backscatter communication systems. It has been shown recently that it is feasible to build backscatter systems supporting QAM using node load selection methodology $[17,27]$. However, improved spectral efficiency means that the node's impedance is mismatched and the power harvested or the efficiency of impedance transformation network $\eta_{2}$ is reduced.

What is more, $m(t)$ can be given as

$$
m(t)=\sum_{k=-\infty}^{\infty} a_{k} g(t-k T),
$$

where $g(t)$ is the rectangle pulse with width $T$ and $\left\{a_{k}\right\}$ is the sequence of symbols with $L$ levels. If the number of phases $\varphi$ is $K$ in (12), the number of the carrier states is $K \times L$. And when $K=L=2$, the modulation is 4QAM, that is, to design and implement 4QAM backscatter modulator, four RC lumped impedances are connected to an antenna port through RF 4-1 Mux, each lumped impedance corresponding to different reflection coefficients. The design of 4QAM colocated backscatter communication systems with this procedure is given in Figure 3. In Figure 3, serial/ parallel converts sensor data into 2 bits for controlling the 4-1 Mux.

To make the structure of the backscatter multisensor nodes simple, we put forward an access scheme with different subcarrier modulations for each sensor; namely, each sensor has different pulse width. So, the cumulative colocated QAM multisensor backscatter signal at the transceivers or reader is a sum of all complex signals from sensors with additive white noise, resulting in

$$
\begin{aligned}
r(t)= & \sqrt{2 P_{i} \alpha_{i}} m_{i}(t) \exp \left[j\left(\omega_{c} t+\varphi\right)\right] \\
& +\sum_{k \neq i} \sqrt{2 P_{k} \alpha_{k}} m_{k}(t) \exp \left[j\left(\omega_{c} t+\varphi\right)\right]+n(t) .
\end{aligned}
$$

4.2. Throughput Maximization for Colocated QAM Multisensor under the RF Energy-Harvesting Constraints. The goal of power control for passive multisensor random interrogating is to meet some QoS criteria, for example, throughput.

In Figure 3, each sensor has different subcarrier frequencies; however, the information leak of one sensor interferes other sensors. The amount of such interference depends on the power spectrum of the chosen subcarrier modulation and the number of sensors. According to the constraint of the correlated short-range wireless communication spectrum, the power spectrum of QAM modulation of the $i$ th sensor can be approximated as [28]

$$
S(f) \approx \frac{1}{\left(f-f_{i}\right)^{4}},
$$

where $f_{i}=1 / T_{i}$ and $T_{i}$ is the pulse width of the $i$ th sensor data. 
Under the lognormal channel, signal-to-interferenceand-noise ratio (SINR) at the transceivers or reader in Figure 3 is written as

$$
\Gamma_{i}=\frac{2 E_{i} h_{i}}{\left(W_{0} / T_{i}\right)+\sum_{j=1, \neq i}^{N} 2 E_{j} h_{j} S\left(f_{j}-f_{i}\right)}
$$

where $E_{i}$ is the transmitted bit energy, $W_{0}$ is the power spectrum density, $h_{i}=l_{i} \Omega_{i}, l_{i}$ is the path loss, $\Omega_{i}$ is the shadow fading of logarithmic normal distribution, and $\Omega_{i}=e^{x}$. x follows normal distribution $G\left(\mu, \sigma^{2}\right)$.

In this paper, the multiple sensors are colocation and share a transmitter and transceiver, so it can be assumed that $h_{i}=h_{j}$ and $E_{i}=E_{j}$. Consequently, (16) can be rewritten as

$$
\Gamma_{i}=\frac{1}{\Gamma_{N}^{-1}+\Gamma_{I}^{-1}}
$$

where $\Gamma_{N}=2 E_{i} T_{i} \cdot l_{i}^{\gamma_{i}} \cdot 10^{x_{i} / 10} / W_{0}$ and $\Gamma_{I}=\sum_{j=1, \neq i}^{N} 10^{\left(x_{i}-x_{j}\right) / 10}$. $\left(l_{i} / l_{j}\right)^{\gamma} \cdot\left(1 /\left(S\left(f_{j}-f_{i}\right)\right)\right)$.

Now, we characterize the performance of backscatter communication. We start by defining the concept of the throughput.

Definition 1. The data delivery ratio $D_{\mathrm{r}}$ of backscatter communication systems is a ratio of successful data packet received to attempted data packet transmitted.

Definition 2. The throughput $T_{\mathrm{h}}$ of backscatter communication systems is the probability that a packet is successfully received during a data interrogation interval.

For a packet to be received successfully, the SINR at the transceivers or reader must exceed some threshold $\lambda_{\text {th }}$. Using the definitions above, the data delivery ratio $D_{\text {ri }}$ and the throughput $T_{\mathrm{h} i}$ in the access interval $T_{\text {access }}$ of the $i$ th sensor can be written, respectively, as

$$
\begin{aligned}
& D_{\mathrm{r} i}=P\left\{\Gamma_{i} \geq \lambda_{\mathrm{th}}\right\}, \\
& T_{\mathrm{h} i}=\frac{P\left\{\Gamma_{i} \geq \lambda_{\mathrm{th}}\right\}}{T_{\mathrm{access}}} .
\end{aligned}
$$

So, the average data delivery ratio and throughput of the backscatter communication systems can be written as follows:

$$
\begin{aligned}
& D_{\mathrm{r}}=\frac{\sum_{i=1}^{N} D_{\mathrm{r} i}}{N}, \\
& T_{\mathrm{h}}=\frac{\sum_{i=1}^{N} T_{\mathrm{h} i}}{N},
\end{aligned}
$$

where $N$ is the number of sensors.

Generally, obtaining throughput in a closed form is not always possible and remains analytically challenging; because of the cumulative distribution function of random variable, $\Gamma_{i}$ remains difficult. Thanks to nodes colocating, this problem can be solved easily. In this case, $x_{i}=x_{j}, l_{i}=l_{j}$, and (16) can be rewritten as

$$
\Gamma_{i}=\frac{1}{\Gamma_{N}^{-1}+\sum_{j=1, \neq i}^{N} S\left(f_{j}-f_{i}\right)} .
$$

Let

$$
k_{i}=10 \log \left(\frac{W_{0}}{2 E_{i} T_{i} l_{i}^{\gamma_{i}}} \cdot \frac{\lambda_{\mathrm{th}}}{1-\lambda_{\mathrm{th}} \sum_{j=1, \neq i}^{N} S\left(f_{j}-f_{i}\right)}\right),
$$

then,

$$
\begin{aligned}
D_{\mathrm{r} i} & =P\left\{\Gamma_{i} \geq \lambda_{\mathrm{th}}\right\}=P\left\{x_{i} \geq k_{i}\right\} \\
& =1-P\left(x_{i}<k_{i}\right)=1-\int_{-\infty}^{k_{i}} \frac{1}{\sqrt{2 \pi} \sigma_{i}} e^{-\left(x_{i}{ }^{2} / 2 \sigma_{i}{ }^{2}\right)} d x .
\end{aligned}
$$

Hence, the average data throughput $T_{\mathrm{h}}$ can be rewritten as

$$
T_{\mathrm{h}}=\frac{\sum_{i=1}^{N} D_{\mathrm{r} i}}{N T_{\mathrm{access}}}=\frac{\sum_{i=1}^{N}\left(1-\int_{-\infty}^{k_{i}}\left(1 / \sqrt{2 \pi} \sigma_{i}\right) e^{-\left(x_{i}^{2} / 2 \sigma_{i}^{2}\right)} d x\right)}{N T_{\text {access }}} .
$$

Assuming duty cycle $\rho=\bar{T}_{d} /\left(\bar{T}_{\mathrm{c}}+\bar{T}_{d}\right)=\bar{T}_{d} / \bar{T}_{\text {task }}, \rho \in$ $[0,1]$, the nonlinear optimization problems of power control for passive QAM backscatter communication systems with $N$ nodes can be expressed as

$$
\max T_{\mathrm{h}}=\frac{\sum_{i=1}^{N} D_{\mathrm{r} i}}{N T_{\text {access }}}
$$

subject to

$$
\begin{aligned}
B_{0 m}+\eta(1-\rho) \bar{T}_{\text {task }}\left(P-N P_{\mathrm{fc}}\right)-\rho N \bar{T}_{\text {task }} P_{\mathrm{bc}} & \geq 0 \\
B_{0 m}+\eta(1-\rho) \bar{T}_{\text {task }}\left(P-N P_{\mathrm{fc}}\right)-\rho N \bar{T}_{\text {task }} P_{\mathrm{bc}} & \leq B \\
B_{0 m}+\eta(1-\rho) \bar{T}_{\text {task }}\left(P-N P_{\mathrm{fc}}\right) & \geq E_{\text {task }}, \\
B_{0} & \leq B .
\end{aligned}
$$

\section{Numerical Results and Discussions}

We evaluate the performance of the power control using numerical analysis. The analysis includes the impact of $P_{\mathrm{bc}}$ on the throughput and the harvested power.

5.1. Parameters for Numerical Analysis. Referring to the relation between bit error rate and SINR for 4QAM under AWGN channel, the threshold $\lambda_{\text {th }}$ can be chosen as 3.0156 corresponding to the data delivery ratio ranging from 1 to $1 \times 10^{-5}$. Meanwhile, the given transmission data rate is $40 \mathrm{kbps}$, the data packet size is $64 \mathrm{bits}$, and the duration of a data packet transmission is $T_{\mathrm{dm}}=1.6 \mathrm{sec}$. All parameters used are listed in Table 1 [29].

5.2. Impact of $P_{\mathrm{bc}}$ on Throughput $T_{\mathrm{h}}$. In this section, we first investigate the choice of $P_{\mathrm{bc}}$ to achieve maximal throughput. 
TABLe 1: Parameters for numerical analysis.

\begin{tabular}{lc}
\hline Parameter & Value \\
\hline$W_{0}$ & $-1.0779 \mathrm{dBw} / \mathrm{Hz}$ \\
$l_{i}$ & $-1 \mathrm{~dB}$ \\
$\lambda_{\mathrm{th}}$ & 3.0156 \\
$T_{\mathrm{dm}}$ & $1.6 \mathrm{sec}$ \\
$P_{\mathrm{fc}}$ & $68.9 \mathrm{nW}$ \\
$B$ & $0.0125 \mathrm{~J}$ \\
$\eta$ & 0.65 \\
\hline
\end{tabular}

Under the energy condition for node operating in (11) and solving the inequality group, it can be shown that the maximum and minimum access interval $T_{\text {access }}$ is given by

$$
\begin{aligned}
T_{\mathrm{access}}^{\min } & =\frac{\eta T_{d}\left(P-N P_{\mathrm{fc}}\right)+N T_{d} P_{\mathrm{bc}}}{\eta\left(P-N P_{\mathrm{fc}}\right)}, \\
T_{\mathrm{access}}^{\max } & =\frac{\eta T_{d}\left(P-N P_{\mathrm{fc}}\right)+N T_{d} P_{\mathrm{bc}}+B}{\eta\left(P-N P_{\mathrm{fc}}\right)} .
\end{aligned}
$$

The access interval $T_{\text {access }}$ is a function of $P_{\mathrm{bc}}$. When $P=189 \mu W, N=2, N=4$, and $N=8$, the minimum and maximum access intervals of sensor node1 with subcarrier $f_{1}=50000 \mathrm{~Hz}$ are plotted in Figures 4 and 5. The subcarriers of other nodes are $50,002 \mathrm{~Hz}, 50,010 \mathrm{~Hz}, 50,100 \mathrm{~Hz}$, $50,110 \mathrm{~Hz}, 50,120 \mathrm{~Hz}, 50,150 \mathrm{~Hz}$, and $50,200 \mathrm{~Hz}$ (the same below). According to these frequencies and the value of $T_{\text {access}}$, we can deduce the corresponding value of the throughput using (20), (23), and (24). And the throughput changes by varying of $T_{\text {access }}$.

It is observed that the maximum and minimum access intervals $T_{\text {access }}$ increase with $P_{\mathrm{bc}}$ in Figures 4 and 5. It is easy to understand, because the greater the $P_{\mathrm{bc}}$, the longer the charge time to meet the energy condition for node operating. It is worthwhile to note that the maximum and minimum access interval $T_{\text {access }}$ increases more with the increase of $N$.

Hence, the maximum throughput as a function of $P_{\mathrm{bc}}$ is plotted under the condition of the minimum access interval $T_{\text {access }}$ in Figure 6 . It is found that the maximal throughput decreases with the increase of $P_{\mathrm{bc}}$. And the maximum throughput is decreased quickly with the increase of $N$.

5.3. Impact of Duty Cycle $\rho$ on Throughput $T_{\mathrm{h}}$. Next, we investigate the impact of varying duty cycle $\rho$ on the throughput $T_{\mathrm{h}}$.

Similar to Section 4.2, the maximum and minimum duty cycle $\rho$ under the energy condition for node operating is given as follows:

$$
\begin{aligned}
\rho_{\max } & =\frac{\eta T_{d}\left(P-N P_{\mathrm{fc}}\right)}{\eta T_{d}\left(P-N P_{\mathrm{fc}}\right)+N T_{d} P_{\mathrm{bc}}}, \\
\rho_{\min } & =\frac{\eta T_{d}\left(P-N P_{\mathrm{fc}}\right)}{B+\eta T_{d}\left(P-N P_{\mathrm{fc}}\right)+N T_{d} P_{\mathrm{bc}}} .
\end{aligned}
$$

For a given $P_{\mathrm{bc}}=0.524 \mu \mathrm{W}$ and a given $P_{\mathrm{fc}}=0.0689 \mu \mathrm{W}$, the throughput $T_{\mathrm{h}}$ as a function of duty cycle $\rho$ is shown in

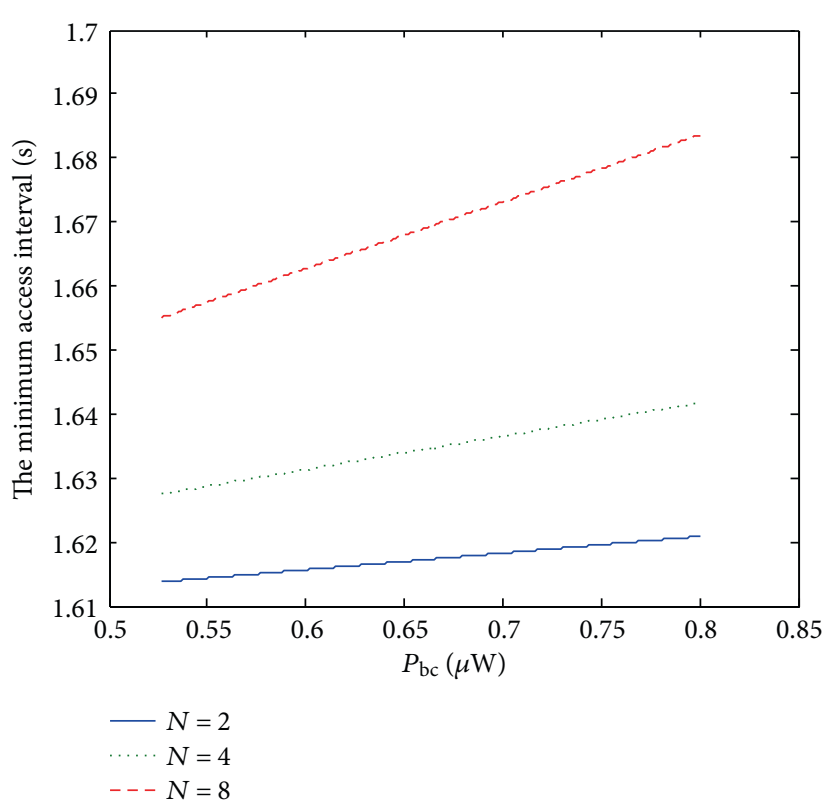

FIGURE 4: The minimum access interval versus the consumed power by sensor with scatter link.

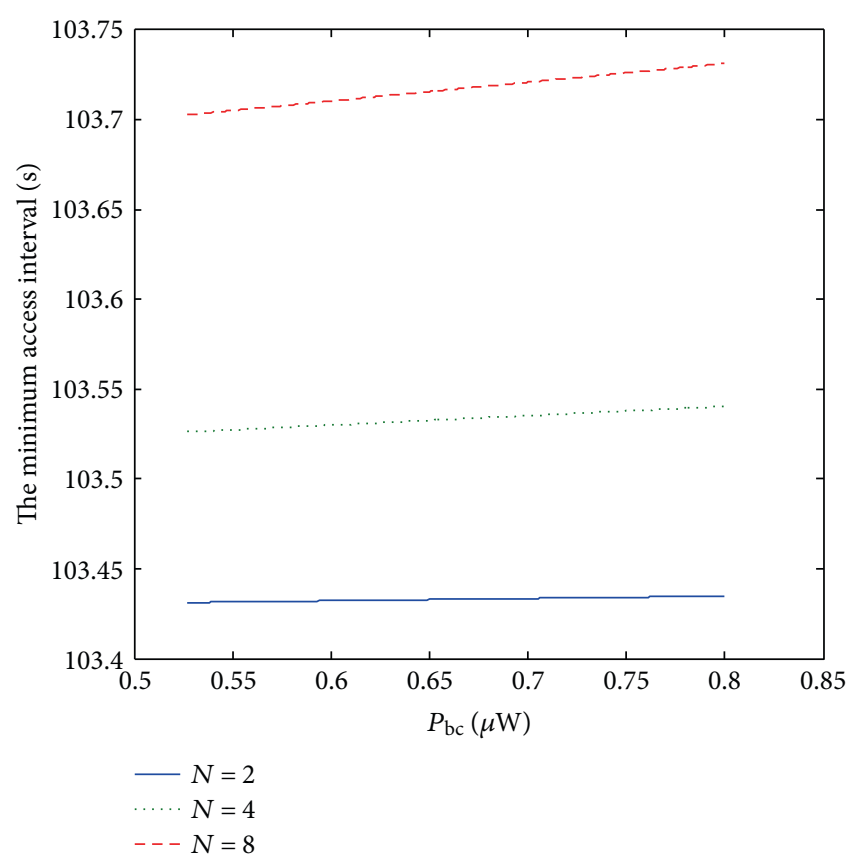

FIgURE 5: The maximum access interval versus the consumed power by sensor with scatter link.

Figure 7. It can be seen that the throughput $T_{\mathrm{h}}$ decreases with the increase of duty cycle $\rho$. Because decreasing duty cycle $\rho$ leads to the increasing of the access interval $T_{\text {access }}$.

It should be noted that $N$ has little effect on the throughput in Figure 7, because duty cycle $\rho$ is insensitive to $N$. Especially, $N$ has almost no effect on the minimum duty cycle $\rho$. We can analyze this using parameter sensitivity. 


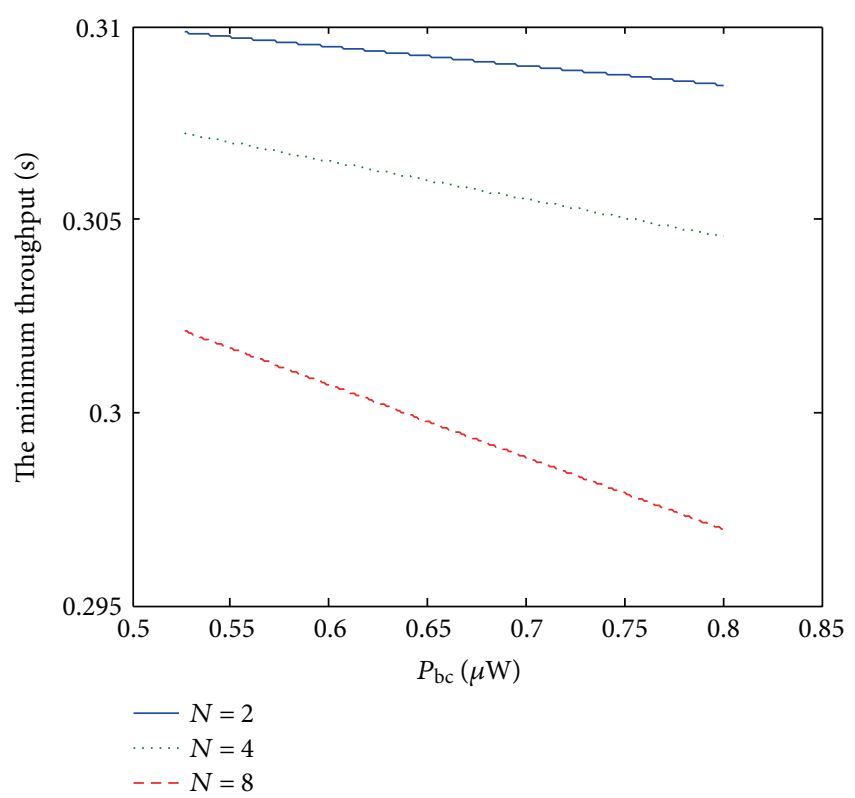

FIGURE 6: The maximum throughput versus the consumed power by sensor with scatter link.

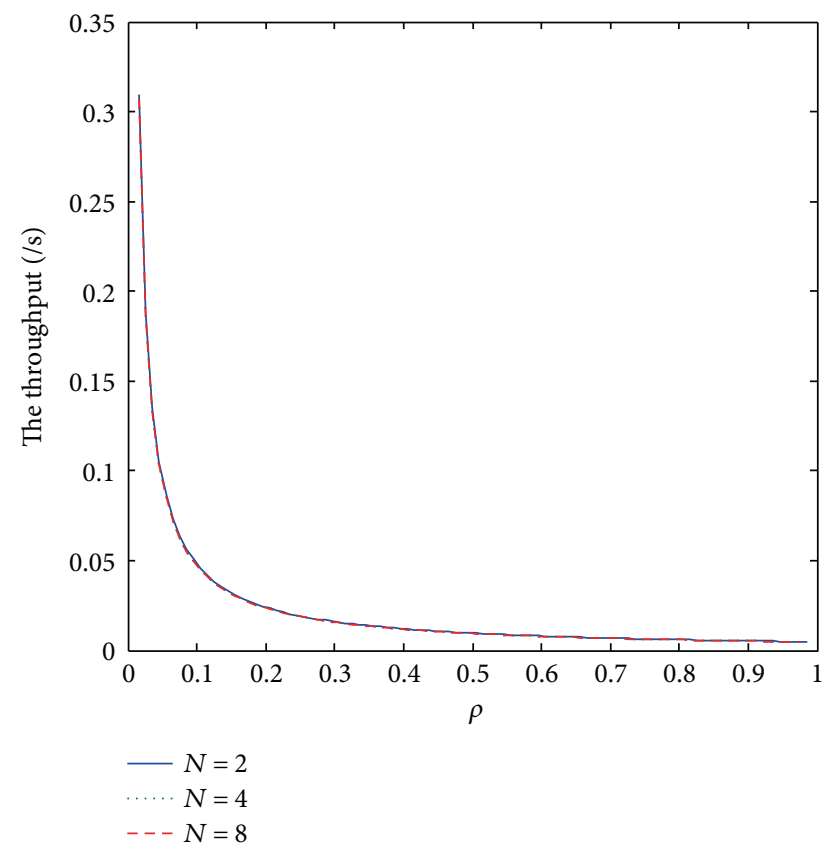

Figure 7: The throughput versus the duty cycle $\rho$.

Taking the partial derivative, the sensitivity of $\rho_{\max }$ and $\rho_{\min }$ is given as

$$
\begin{aligned}
& S_{\rho_{\max }}=\left|\frac{\partial \rho_{\max }}{\partial N}\right|=\frac{\eta T_{d}^{2} P P_{\mathrm{bc}}}{\left[\eta T_{d}\left(P-N P_{\mathrm{fc}}\right)+N T_{d} P_{\mathrm{bc}}\right]^{2}}, \\
& S_{\rho_{\min }}=\left|\frac{\partial \rho_{\min }}{\partial N}\right|=\frac{\eta\left(T_{d}^{2} P P_{\mathrm{bc}}+T_{d} B P_{\mathrm{fc}}\right)}{\left[B+\eta T_{d}\left(P-N P_{\mathrm{fc}}\right)+N T_{d} P_{\mathrm{bc}}\right]^{2}} .
\end{aligned}
$$

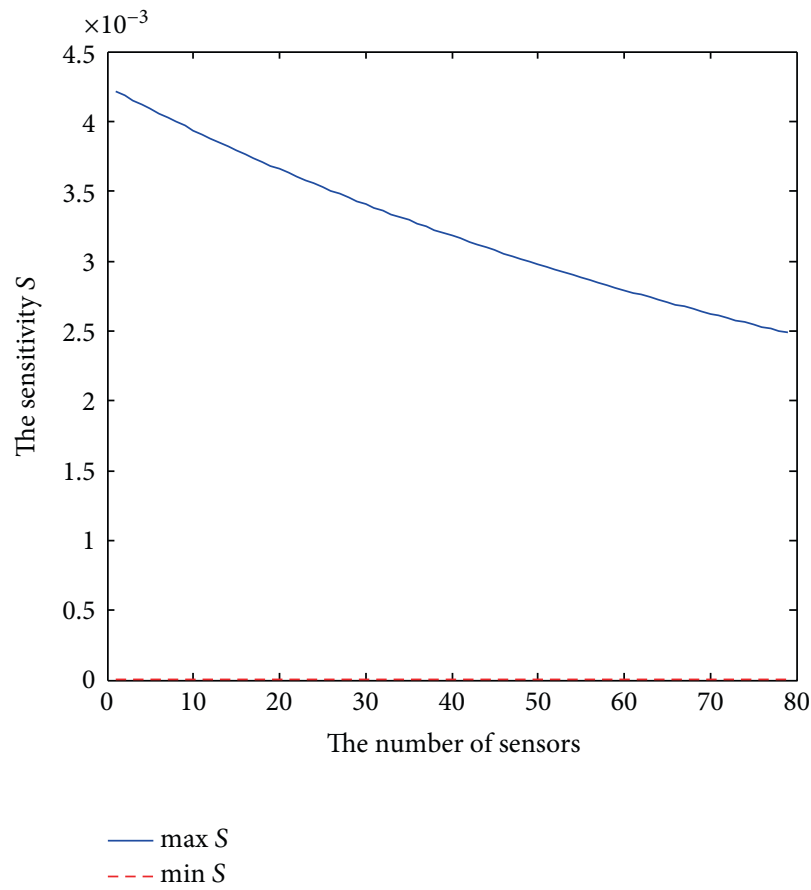

FIgURE 8: The sensitivity of duty cycle about $N$.

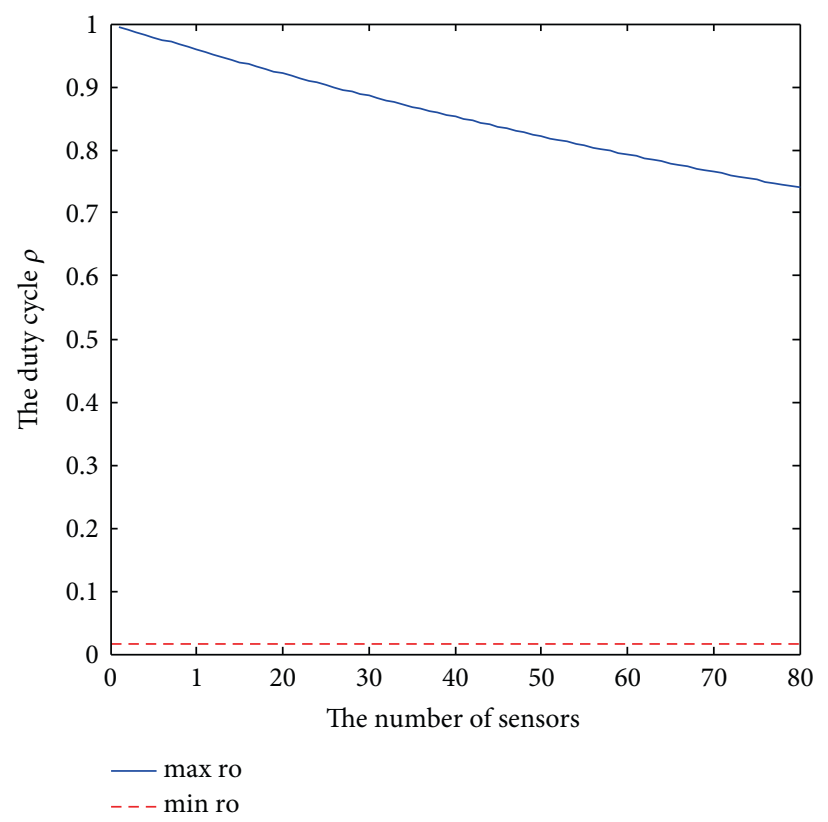

Figure 9: The relation between the duty cycle $\rho$ and $N$.

Hence, the $S_{\rho_{\max }}$ and $S_{\rho_{\min }}$ as functions of $N$ are shown in Figure 8. In Figure $8, S_{\rho_{\max }}$ and $S_{\rho_{\min }}$ are very small. Especially, $N$ has almost no effect on the minimum duty cycle $\rho$, because of using the large stored capacity. This can be also seen from the relation between duty cycle $\rho$ and $N$ shown in Figure 9.

5.4. Impact of $P_{\mathrm{bc}}$ on the Minimum Harvested Energy $P_{\min }$. Finally, we analyze the choice of $P_{\mathrm{bc}}$ to achieve 


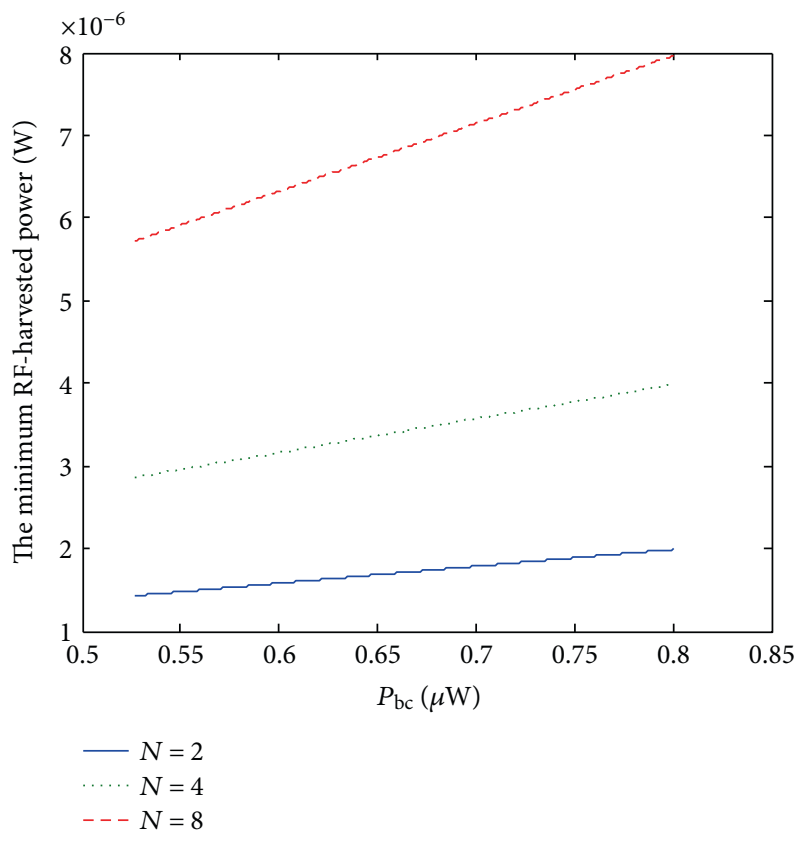

Figure 10: The minimum RF-harvested power versus the consumed power by sensor with scatter link.

the minimal RF-harvested energy under the energy condition for node operating.

In the same way, we can derive that the minimal RF-harvested energy is given by

$$
P_{\min }=N P_{\mathrm{bc}}+\frac{E_{\mathrm{task}}}{\eta(1-\rho) T_{\mathrm{task}}} .
$$

The $P_{\min }$ as a function of $P_{\mathrm{bc}}$ is plotted in Figure 10. In Figure 10, as the $P_{\mathrm{bc}}$ and $N$ increase, the $P_{\min }$ increases. This conclusion is obvious. Similar to Section 5.2, the number of sensors has larger effect on the minimum RFharvested power.

\section{Conclusion}

To achieve good QoS level such as throughput, power control is of great importance to passive QAM multisensor backscatter communication systems. This paper presents the RF energy-harvesting model and gives the energy condition for node operating. In order to minimize interference resulted from multisensors and increase spectral efficiency, we propose the colocated passive QAM backscatter communication signal model, give the closed-form solution of the throughput under log-normal channel, and put forward the nonlinear optimization problems of power control for passive QAM backscatter communication systems. Solving the nonlinear optimization problems, we obtain the maximum and minimum access intervals, the maximum and minimum duty cycle, and the minimal RF harvested energy under the condition of node operating. Based on the solutions above, we analyze the maximum throughput of passive QAM backscatter communication systems and make numerical calculation. Finally, we make the conclusion as follows:

(i) The energy condition for node operating is a prerequisite for designing and optimizing the passive backscatter communication systems, and the goal of power control is to maximize the QoS of the systems.

(ii) Under the energy condition for node operating, the consumed power $P_{\mathrm{bc}}$ of the sensor with backscatter link and the number of sensors has large effect on the throughput of systems. It is found that the maximal throughput decreases with the increase of $P_{\mathrm{bc}}$ and $N$ and the maximum throughput is decreased quickly with the increase of $N$.

(iii) Especially, for a given $P_{\mathrm{bc}}$ and a given $P_{\mathrm{fc}}$, it can be seen that the throughput $T_{\mathrm{h}}$ decreases with the increase of duty cycle $\rho$ and the number of sensors has little effect on the throughput.

(iv) There also exists a further improvement in this paper. For the next phase work, we will try to establish an actual model for data measurement and we will consider the WCDMA or LTE signals substituting for QAM signals.

\section{Conflicts of Interest}

The authors declare that they have no conflicts of interest.

\section{Acknowledgments}

This research is a project partially supported by the National Natural Science Foundation of China (Grant no. 61362004) and Guizhou Joint Natural Science Foundation (Grant no. LKS [2013] 25).

\section{References}

[1] H. Stockman, "Communication by means of reflected power," Proceedings of the IRE, vol. 36, no. 10, pp. 1196-1204, 1948.

[2] A. Sample, D. Yeager, and P. Powledge, "Design of an RFIDbased battery-free programmable sensing platform," IEEE Transactions on Instrumentation and Measurement, vol. 57, no. 11, pp. 2608-2615, 2008.

[3] D. Yeager, F. Zhang, and A. Zarrasvand, "A $9 \mu \mathrm{A}$, addressable Gen2 sensor tag for biosignal acquisition," IEEE Journal of Solid-State Circuits, vol. 45, no. 10, pp. 2198-2209, 2010.

[4] W. U. Bajwa, A. Sayeed, and R. Nowak, "Matched sourcechannel communication for field estimation in wireless sensor networks," in Proceedings of Information Processing in Sensor Networks, 2005, Los Angeles, CA, USA, April 2005.

[5] S. Ergen, A. S. Vincentelli, and X. Sun, "The tire as an intelligent sensor," IEEE Transaction on Computer-Aided Design of Integrated Circuits and Systems, vol. 28, no. 7, pp. 941955, 2009.

[6] J. Grosinger, "Feasibility of backscatter RFID systems on the human body," EURASIP Journal on Embedded Systems, vol. 2013, no. 1, pp. 1-10, 2013. 
[7] S. Hu, S. Bing, and S. Heng, "Power transmission performance for ultra high frequency embedded RFID system in tire," Transactions of the Chinese Society of Agricultural Engineering, vol. 29, no. 21, pp. 150-158, 2013.

[8] P. V. Bharat, N. Sihna, and K. E. Pujitha, "Tire pressure monitoring system using ambient backscatter technology containing RF harvesting circuitry," International Journal of Advance Engineering and Research Development, vol. 1, no. 6, pp. 1-11, 2014.

[9] P. Ferrari, A. Flammini, and E. Sisinni, "New architecture for a wireless smart sensor based on a software defined radio," IEEE Transaction on Instrumentation and Measurement, vol. 60, no. 6, pp. 2133-2141, 2011.

[10] A. Kansal, D. Potter, and M. B. Srivastava, "Performance aware tasking for environmentally powered sensor networks," in Proceedings of ACM SIGMETRICS, vol. 32, no. 1, pp. 223234, New York, NY, USA, 2006.

[11] H. Yoo, M. Shim, and D. Kim, "Self-synchronized and energyaware duty cycle control algorithm in energy-harvested wireless sensor networks," IEEE Communications Letters, vol. 16, no. 2, pp. 203-204, 2012.

[12] L. Varga, G. Romaniello, and M. Favre, "GreenNet: an energyharvesting IP-enabled wireless sensor network," IEEE Internet of Things Journal, vol. 2, no. 5, pp. 412-426, 2015.

[13] A. Kansal, J. Hsu, and S. Zahedi, "Power management in energy harvesting sensor networks," ACM Transactions on Embedded Computing Systems, vol. 6, no. 4, pp. 1-35, 2007.

[14] M. Z. Win, P. C. Pinto, and L. A. Shepp, "A mathematical theory of network interference and its applications," Proceedings of the IEEE, vol. 97, no. 2, pp. 205-230, 2009.

[15] A. Bletsas, A. G. Dimitriou, and J. N. Sahalos, "Improving backscatter radio tag efficiency," IEEE Transaction on Microwave Theory and Techniques, vol. 58, no. 6, pp. 1502-1509, 2010.

[16] H. Ju and R. Zhang, "Throughput maximization in wireless powered communication networks," IEEE Transactions on Wireless Communications, vol. 13, no. 1, pp. 418-428, 2014.

[17] S. J. Thomas, E. Wheeler, and J. Teizer, "Quadrature amplitude modulated backscatter in passive and semipassive UHF RFID systems," IEEE Transaction on Microwave Theory and Techniques, vol. 60, no. 4, pp. 1175-1182, 2012.

[18] G. Papotto, F. Carrara, A. Finocchiaro, and G. Palmisano, "A 90-nm CMOS 5-mbps crystal-less RF-powered transceiver for wireless sensor network nodes," IEEE Journal of SolidState Circuits, vol. 49, no. 2, pp. 335-346, 2014.

[19] C. Ok, H. Thadakamalla, and U. Raghavan, "Optimal transmission power in self-sustainable sensor networks for pipeline monitoring," in 2007 IEEE International Conference on Automation Science and Engineering, Scottsdale, AZ, USA, 2007.

[20] X. Lu, P. Wang, D. Niyato, D. I. Kim, and Z. Han, "Wireless networks with RF energy harvesting: a contemporary survey," Networking and Internet Architecture, 2014, http://arxiv.org/ abs/1406.6470.

[21] L. Xia, J. Cheng, N. E. Glover, and P. Chiang, " 0.56 V, $-20 \mathrm{dBm}$ RF-powered, multi-node wireless body area network systemon-a-chip with harvesting-efficiency tracking loop," IEEE Journal of Solid-State Circuits, vol. 49, no. 6, pp. 13451355, 2014.

[22] S. Agrawal, S. K. Pandey, J. Singh, and M. S. Parihar, "Realization of efficient RF energy harvesting circuits employing different matching technique," in 2014 15th International Symposium on Quality Electronic Design (ISQED), pp. 754761, Santa Clara, CA, USA, March, 2014.

[23] S. Leng, D. W. K. Ng, and R. Schober, "Power efficient and secure multiuser communication systems with wireless information and power transfer," in 2014 IEEE International Conference on Communications Workshops (ICC), Sydney, Australia, June, 2014.

[24] A. Seyedi and B. Sikda, "Energy efficient transmission strategies for body sensor networks with energy harvesting," Transactions on Communications, vol. 58, no. 7, pp. 21162126, 2010.

[25] H. P. Tan, P. W. Lee, W. K. Seah, and Z. A. Eu, "Impact of power control in wireless sensor networks powered by ambient energy harvesting (WSN-HEAP) for railroad health monitoring," in WAINA '09. International Conference on Advanced Information Networking and Applications Workshops, Bradford, UK, May 2009.

[26] D. M. Dobkin, The RF in RFID: Passive UHF RFID in Practice, Burlington, MA: Newnes, USA, 2008.

[27] T. S. Ajitha and V. Bhanumathi, "Improving RFID tag efficiency by QAM backscatter modulation," International Journal of Scientific \& Engineering Research, vol. 4, no. 4, pp. 421-427, 2013.

[28] L. Burgess, "FCC and ETSI requirements for short-range UHF ASK modulated transmitters," High Frequency Electronics, vol. 12, pp. 28-35, 2005.

[29] R. Gonsalves, "Evaluation of outage probability in two-tier open access femtocell networks," in 2014 International Conference on Circuits, Systems, Communication and Information Technology Applications (CSCITA), Mumbai, India, April 2014. 


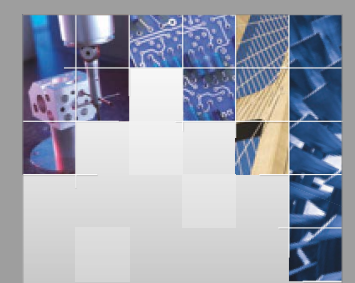

\section{Enfincering}
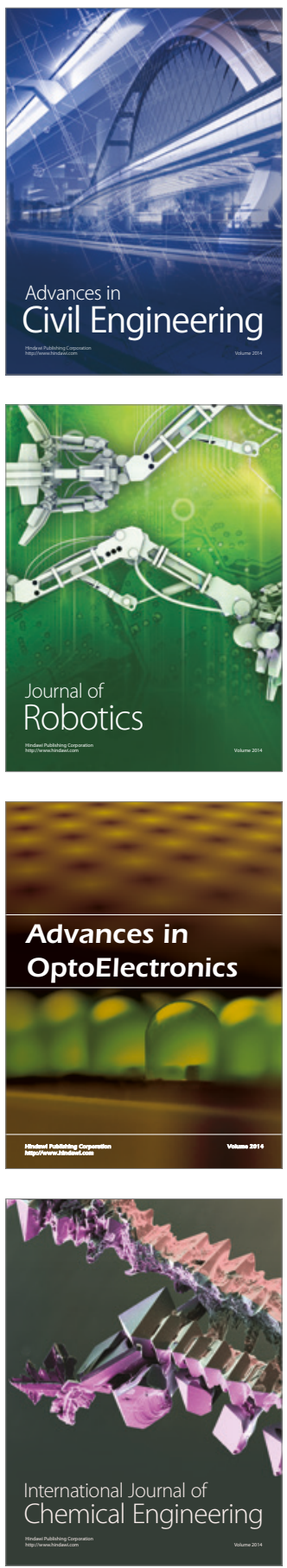

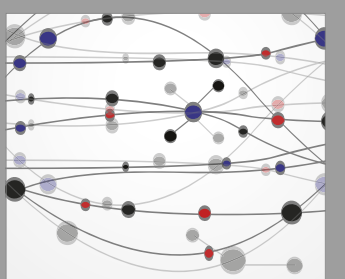

The Scientific World Journal

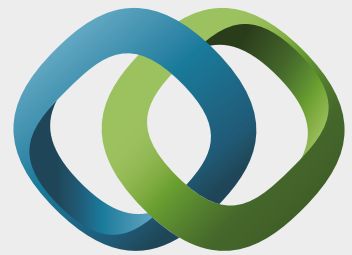

\section{Hindawi}

Submit your manuscripts at

https://www.hindawi.com
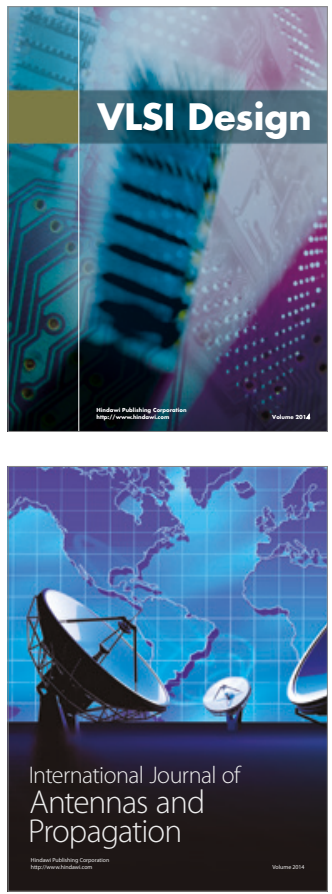

\section{Rotating}

Machinery
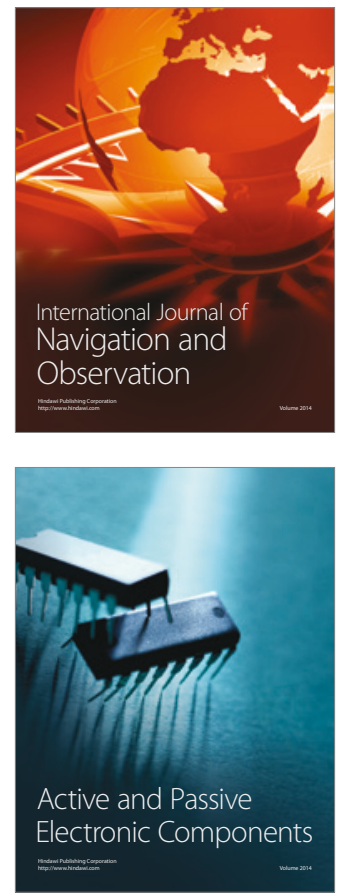
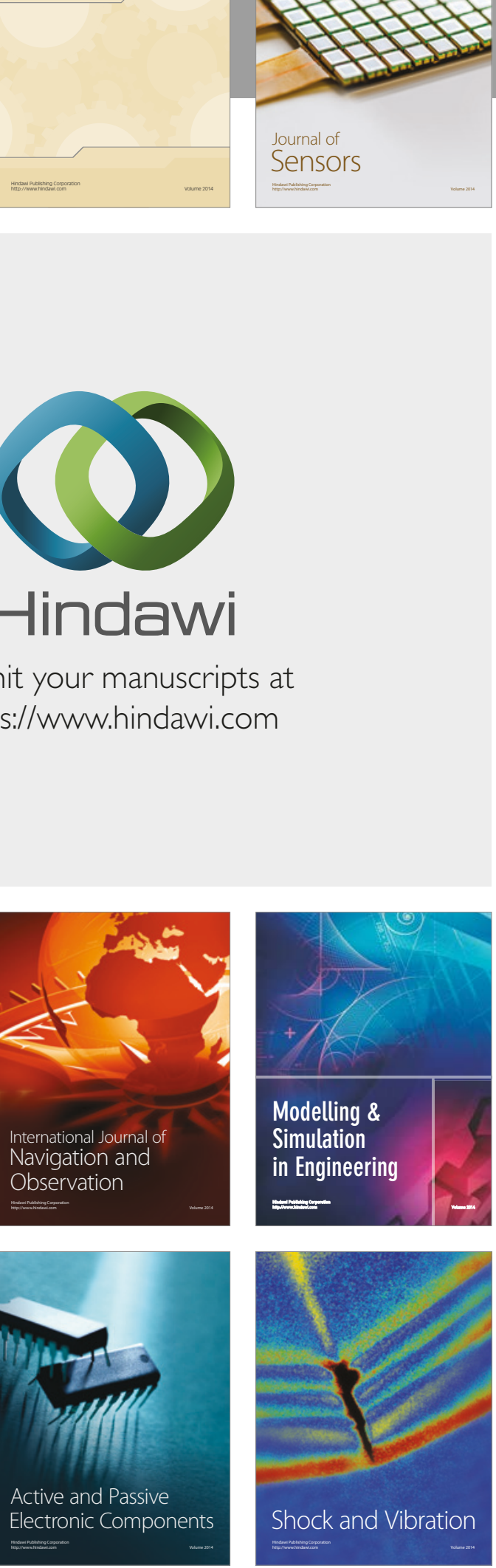
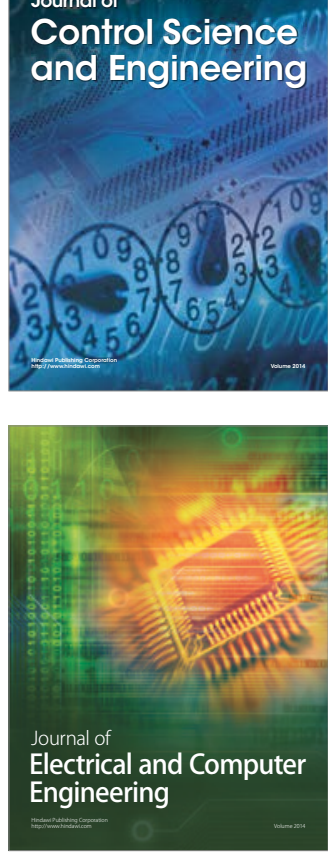

Distributed

Journal of

Control Science

and Engineering
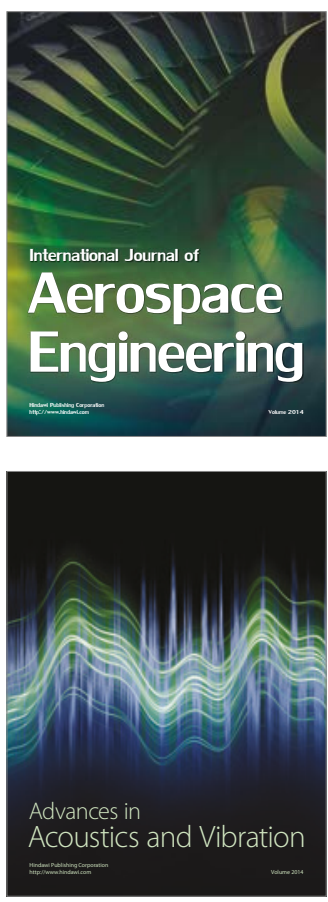

Sensor Networks 